\title{
Theoretical Description of RESPIRATION-CP
}

\author{
Anders B. Nielsen, ${ }^{1,2}$ Kong Ooi Tan, ${ }^{2}$ Ravi Shankar, ${ }^{1}$ Susanne Penzel, ${ }^{2}$ Riccardo \\ Cadalbert $^{2}$, Ago Samoson ${ }^{3}$, Beat H. Meier, ${ }^{2}$ and Matthias Ernst ${ }^{2}$
}

${ }^{1}$ Center for Insoluble Protein Structures (inSPIN), Interdisciplinary Nanoscience Center (iNANO) and Department of Chemistry, Aarhus University, Gustav Wieds Vej 14, DK-8000 Aarhus C, Denmark. ${ }^{2}$ Laboratory of Physical Chemistry, ETH Zürich, Vladimir-Prelog-Weg 2, 8093 Zürich, Switzerland.

${ }^{3}$ NMR Instituut, Tartu Teaduspark, Tehnomeedikum, Tallinn University of Technology, Akadeemia tee 15a, 19086, Tallinn, Estonia.

Corresponding authors: Anders B. Nielsen (abn@inano.au.dk) and Matthias Ernst (maer@phys.chem.ethz.ch) 
We present a quintuple-mode operator-based Floquet approach to describe arbitrary amplitude modulated cross polarization experiments under magic-angle spinning (MAS). The description is used to analyze variants of the RESPIRATION approach $\left({ }^{\mathrm{RESPIRATION}} \mathrm{CP}\right)$ where recoupling conditions and the corresponding first-order effective Hamiltonians are calculated, validated numerically and compared to experimental results for ${ }^{15} \mathrm{~N}-{ }^{13} \mathrm{C}$ coherence transfer for uniformly ${ }^{13} \mathrm{C},{ }^{15} \mathrm{~N}$-labeled alanine and for uniformly ${ }^{2} \mathrm{H},{ }^{13} \mathrm{C},{ }^{15} \mathrm{~N}$-labeled (deuterated and $100 \%$ back-exchanged) ubiquitin at spinning frequencies of 16.7 and $90.9 \mathrm{kHz}$. Similarities and differences between different implementations of the ${ }^{\mathrm{RESPIRATION}} \mathrm{CP}$ sequence using either $\mathrm{CW}$ irradiation or small flip-angle pulses are discussed.

KEYWORDS Solid-State NMR, Floquet Theory, dipolar recoupling, Cross Polarization (CP), RESPIRATION-CP 


\section{Introduction}

Heteronuclear dipolar interactions are used for polarization transfer for solid-state NMR experiments and for extracting information about internuclear distances, dihedral angles, and order parameters, which are important for the determination of structure and dynamics of biomolecules with atomic resolution $^{1-6}$. Dipolar interactions are averaged out by magic-angle spinning (MAS) but may be reintroduced by recoupling sequences that rely on interference between spin and space rotations ${ }^{7-10}$.

An example of a widely used heteronuclear dipolar recoupling sequence is the Hartmann-Hahn cross-polarization $(\mathrm{CP})$ experiment ${ }^{11-13}$. Polarization transfer between high- $\gamma$ and low- $\gamma\left(\right.$ e.g., ${ }^{1} \mathrm{H}_{-}{ }^{13} \mathrm{C}$ or ${ }^{1} \mathrm{H}_{-}{ }^{15} \mathrm{~N}$ ) nuclei are the starting point of many solid-state NMR experiment and an element of the 3D pulse sequences used for protein assignment like NCOCA, NCACO, CANCO, NCACB rely on this element for one or more transfer $\operatorname{steps}^{14,15}$. As the sequence is often used several times in a multidimensional experiment, it is important that each transfer step is as efficient as possible.

A standard $\mathrm{CP}$ experiment consists of simultaneous continuous-wave $(\mathrm{CW})$ irradiation on the two nuclei of interest. The rf amplitudes are matched to satisfy the modified Hartmann-Hahn condition ${ }^{12}$ under MAS, i.e., $\omega_{1 \mathrm{I}} \pm \omega_{1 \mathrm{~S}}=n \omega_{\mathrm{r}}$ where $n$ can take the values of \pm 1 and \pm 2 . In order to reduce the sensitivity towards mismatch of the rf fields and rf-field inhomogeneity as well as chemical-shift offsets, several modifications of the standard Hartmann-Hahn cross-polarization experiment are described in the literature ${ }^{16-20}$. One of these modifications is the Rotor Echo Short Pulse IRrAdiaTION mediated cross polarization $\left({ }^{\mathrm{RESPIRATION}} \mathrm{CP}\right)^{21-25}$. This experiment consists of rotor-synchronized short pulses on both channels and an amplitude-modulated rf irradiation between the short pulses on one of the channels. This setup has been proven to be efficient, in particular, to transfer polarization from spin- 1 nuclei $\left({ }^{2} \mathrm{H},{ }^{14} \mathrm{~N}\right)$ to spin- $1 / 2$ nuclei $\left({ }^{13} \mathrm{C}\right)^{24,}{ }^{25}$. Compared to standard Hartmann-Hahn cross polarization, the short pulses on the ${ }^{2} \mathrm{H}$ channel were shown to be more efficient in terms of transfer efficiency to ${ }^{13} \mathrm{C}$ nuclei. They also lead to a more uniform polarization transfer over a large range of quadrupolar-coupling constants ${ }^{24}$. 
The first-order effective Hamiltonian for the ${ }^{\text {RESPIRATION }} \mathrm{CP}$ experiment has already been calculated using average-Hamiltonian theory $(\mathrm{AHT})^{26}$. However, such an analysis has limitation, for instance, it is difficult to generalize to different regimes of rf-field amplitudes and MAS frequencies. This is a consequence of the fact that AHT is limited to stroboscopic observation and synchronization of the different time-dependent processes. On the other hand, Floquet theory allows the treatment of timedependent Hamiltonians with multiple incommensurate frequencies without making assumptions about the relative magnitudes of the time scales ${ }^{27-30}$.

In this article, we provide a theoretical description for arbitrary amplitude-modulated crosspolarization experiment that is characterized by five frequencies, and, therefore, a quintuple-mode Floquet framework is needed to analyze such sequences. Such a framework allows the determination of resonance conditions and the corresponding effective Hamiltonians. By using this description, we analyze the ${ }^{\text {RESPIRATION }} \mathrm{CP}$ experiment where one can gain insights into the effect of finite pulses, which are not negligible in the fast MAS regime $\left(v_{\mathrm{r}} \approx 100 \mathrm{kHz}\right)$. We also show that ${ }^{\text {RESPIRATION }}{ }^{\mathrm{CP}}$ can either be implemented using the original short pulses or in a different way where the short pulses are replaced by CW irradiation. Finally, it will be shown that the effective Hamiltonians for the two implementations show differences even though both sequences have very similar resonance conditions and a comparison to the standard Hartmann-Hahn CP experiment is given.

\section{Theory}

Let us consider a two-spin system consisting of an I and S spin with isotropic and anisotropic chemical shielding which are coupled by $J$ and dipolar interaction. In the usual rotating frame the timedependent Hamiltonian of the system under MAS and rf irradiation is given by

$$
\hat{H}(t)=\omega_{\mathrm{I}}(t) \hat{I}_{z}+\omega_{\mathrm{S}}(t) \hat{S}_{z}+\omega_{\mathrm{IS}}(t) 2 \hat{I}_{z} \hat{S}_{z}+\pi J_{\mathrm{IS}} 2 \hat{I}_{z} \hat{S}_{z}+\hat{H}_{\mathrm{rf}}(t)
$$

where $\omega_{\mathrm{I}}(t), \omega_{\mathrm{S}}(t)$, and $\omega_{\mathrm{IS}}(t)$ are the time-dependent chemical shifts for I- and S-spin and the dipolar coupling, respectively, and $J_{\text {IS }}$ denotes the scalar coupling. In general, all time-dependent interactions 
can be expressed as Fourier series $\omega_{\Lambda}(t)=\sum_{n=-2}^{2} \omega_{\Lambda}^{(n)} e^{i n \omega_{\mathrm{r}} t}$ where $\omega_{\mathrm{r}}$ denotes the spinning frequency and $\Lambda=I, S, I S$. For the ${ }^{\text {RESPIRATION }} \mathrm{CP}$ pulse-sequence element depicted in Fig $1 \mathrm{~A}$, the time-dependent rffield Hamiltonian can be described by a pure amplitude modulation as

$$
\hat{H}_{\mathrm{rf}}(t)=\omega_{1}^{(\mathrm{I})}(t) \hat{I}_{x}+\omega_{1}^{(\mathrm{S})}(t) \hat{S}_{x}
$$

where $\omega_{1}^{(\mathrm{I})}(t)$ and $\omega_{1}^{(\mathrm{S})}(t)$ denote the time-dependent rf-field amplitudes on $\mathrm{I}$ and $\mathrm{S}$ channel, respectively.

\section{Figure 1}

For any amplitude-modulated rf irradiation, the rf-field Hamiltonian of Eq. (2) can be separated into an average, time-independent part $\omega_{1, \mathrm{CW}}^{(\Lambda)}=\overline{\omega_{1}^{(\Lambda)}(t)}$ where $\Lambda$ denotes either I- or S-channel and a timedependent component with zero net nutation angle $\omega_{1, \mathrm{~m}}^{(\Lambda)}(t)=\omega_{1}^{(\Lambda)}(t)-\omega_{1, \mathrm{CW}}^{(\Lambda)}$ which need to be treated separately in the interaction-frame transformation. This separation leads to a rf-field Hamiltonian of the form:

$$
\hat{H}_{\mathrm{rf}}(t)=\left(\omega_{1, \mathrm{CW}}^{(\mathrm{I})}+\omega_{1, \mathrm{~m}}^{(\mathrm{I})}(t)\right) \hat{I}_{x}+\left(\omega_{1, \mathrm{CW}}^{(\mathrm{S})}+\omega_{1, \mathrm{~m}}^{(\mathrm{S})}(t)\right) \hat{S}_{x}
$$

In the case of ${ }^{\text {RESPIRATION }} \mathrm{CP}$, this separation is presented in Fig. 1A. The amplitude of the CW field depends only on the effective flip angle of the short pulse and is given by $\omega_{1, \mathrm{CW}}^{(\Lambda)}=\omega_{1}^{(\Lambda)} \tau_{\mathrm{p}}^{(\Lambda)} / \tau_{\mathrm{m}}^{(\Lambda)}$ where $\tau_{\mathrm{p}}^{(\Lambda)}$ is the pulse length of the short pulse with $\mathrm{rf}$ amplitude $\omega_{1}^{(\Lambda)}$ and $\tau_{\mathrm{m}}^{(\Lambda)}$ is the cycle time on either the I- or S-spin channel. The amplitude modulated part can be expressed by a Fourier series with the basic frequency $\omega_{\mathrm{m}}^{(\Lambda)}=2 \pi / \tau_{\mathrm{m}}^{(\Lambda)}$ as

$$
\omega_{1, \mathrm{~m}}^{(\Lambda)}(t)=\sum_{k=-\infty}^{\infty} \omega_{k}^{(\Lambda)} e^{i k \omega_{\mathrm{m}}^{(\Lambda)} t}
$$


where $\omega_{k}^{(\Lambda)}$ are the Fourier coefficients with $\omega_{0}^{(\Lambda)}=0$. The most general description of ${ }^{\text {RESPIRATION }}$ CP has to take five frequencies into account: the two $\mathrm{CW}$-nutation frequencies $\omega_{1, \mathrm{CW}}^{(\Lambda)}$, two modulation frequencies $\omega_{\mathrm{m}}^{(\Lambda)}$, and the MAS frequency $\omega_{\mathrm{r}}$. In typical implementations, however, the modulation frequencies on the two channels will often be the same.

To use Floquet theory, we have to transform the system Hamiltonian into the full rfinteraction frame. For this we tilt the coordinate system first by $\pi / 2$ about the $-y$ axis and then go into the rf interaction frame which can be described by the transformation:

$$
\hat{U}(t)=e^{-i \beta_{\mathrm{1}}(t) \hat{I}_{z}} e^{-i \beta_{\mathrm{S}}(t) \hat{S}_{z}} e^{-i \frac{\pi}{2}\left(\hat{I}_{y}+\hat{S}_{y}\right)}
$$

with $\beta_{I}(t)$ and $\beta_{S}(t)$ defining the flip angle which is calculated by direct integration of the rf field. The interaction-frame Hamiltonian will then be modulated by functions of the form

$$
e^{i \beta_{\Lambda}(t)}=\sum_{k=-\infty}^{\infty} a_{k}^{(\Lambda)} e^{i k \omega_{\mathrm{m}}^{(\Lambda)} t} e^{i \omega_{1, \mathrm{CW}}^{(\Lambda)}} \text { and } e^{-i \beta_{\Lambda}(t)}=\sum_{k=-\infty}^{\infty}\left(a_{-k}^{(\Lambda)}\right)^{*} e^{i k \omega_{\mathrm{m}}^{(\Lambda)} t} e^{-i \omega_{1, \mathrm{cW}}^{(\Lambda)} t}
$$

where the $a_{k}^{(\Lambda)}$ are the Fourier coefficients of the interaction-frame transformation. The interactionframe Hamiltonian can now be written as a Fourier series with five frequencies as

$$
\hat{\tilde{H}}(t)=\sum_{n=-2}^{2} \sum_{k_{\mathrm{I}}=-\infty}^{\infty} \sum_{k_{\mathrm{S}}=-\infty}^{\infty} \sum_{\ell_{\mathrm{I}}=-1}^{1} \sum_{\ell_{\mathrm{S}}=-1}^{1} \hat{\tilde{H}}^{\left(n, k_{\mathrm{I}}, \ell_{\mathrm{I}}, k_{\mathrm{S}}, \ell_{\mathrm{S}}\right)} e^{i n \omega_{\mathrm{r}} t} e^{i k_{\mathrm{I}} \omega_{\mathrm{m}}^{(\mathrm{I})} t} e^{i \ell_{\mathrm{I}} \omega_{\mathrm{l}, \mathrm{Cw}}^{(\mathrm{I})} t} e^{i k_{\mathrm{S}} \omega_{\mathrm{m}}^{(\mathrm{S})} t} e^{i \ell_{\mathrm{S}} \omega_{1, \mathrm{CW}}^{(\mathrm{S})} t}
$$

In contrast to average Hamiltonian theory (AHT), there is no requirement that the different frequencies are commensurate and that the Hamiltonian is cyclic in the AHT sense ${ }^{31}$. The Fourier components $\hat{\tilde{H}}^{\left(n, k_{\mathrm{I}}, \ell_{1}, k_{\mathrm{s}}, \ell_{\mathrm{S}}\right)}$ are given by

$$
\hat{\tilde{H}}^{(n, 0,0,0,0)}=0
$$




$$
\begin{gathered}
\hat{\tilde{H}}^{\left(n, k_{1}, \pm 1,0,0\right)}=-\omega_{\mathrm{I}}^{(n)} \frac{1}{2} \hat{I}^{ \pm} f\left(a_{k_{\mathrm{I}}}^{(\mathrm{I})}, \pm 1\right) \\
\hat{\tilde{H}}^{\left(n, 0,0, k_{\mathrm{s}}, \pm 1\right)}=-\omega_{\mathrm{S}}^{(n)} \frac{1}{2} \hat{S}^{ \pm} f\left(a_{k_{\mathrm{S}}}^{(\mathrm{S})}, \pm 1\right) \\
\hat{\tilde{H}}^{\left(n, k_{\mathrm{l}}, \pm 1, k_{\mathrm{s}}, \pm 1\right)}=\frac{1}{2} \omega_{\mathrm{IS}}^{(n)} \hat{I}^{ \pm} \hat{S}^{ \pm} f\left(a_{k_{l}}^{(\mathrm{I})}, \pm 1\right) f\left(a_{k_{\mathrm{S}}}^{(\mathrm{S})}, \pm 1\right) .
\end{gathered}
$$

The function

$$
f\left(a_{k}^{(\Lambda)}, z\right)=\left\{\begin{array}{cc}
a_{k}^{(\Lambda)} & \text { for } z=+1 \\
\left(a_{-k}^{(\Lambda)}\right)^{*} & \text { for } z=-1
\end{array}\right.
$$

selects the conjugate complex value for $a_{k}^{(\Lambda)}$ and the negative sign of the index $k$ for $z=-1$. The $\omega_{\Lambda}^{(0)}$ terms correspond to the isotropic interaction, i.e., $J$ coupling $\left(\omega_{I S}^{(0)}=\pi J\right)$ and isotropic chemical shifts.

For any amplitude modulated cross polarization experiment, we find the resonance conditions based on the interaction-frame Hamiltonian from Eq. (5) to be given by

$$
n \omega_{\mathrm{r}}+k_{\mathrm{I}} \omega_{\mathrm{m}}^{(\mathrm{I})}+\ell_{\mathrm{I}} \omega_{1, \mathrm{CW}}^{(\mathrm{I})}+k_{\mathrm{S}} \omega_{\mathrm{m}}^{(\mathrm{S})}+\ell_{\mathrm{S}} \omega_{1, \mathrm{CW}}^{(\mathrm{S})}=0
$$

where $n, k_{\mathrm{I}}, k_{\mathrm{S}}, \ell_{\mathrm{I}}$, and $\ell_{\mathrm{S}}$ are integers. The values of $n$ are restricted to \pm 1 and $\pm 2, \ell_{\Lambda}$ to \pm 1 (in the absence of homonuclear couplings), while $k_{\Lambda}$ can take any integer value. The corresponding first-order effective Hamiltonian obtained from Floquet theory is then given by

$$
\hat{\bar{H}}^{(1)}=\hat{\tilde{H}}^{(0,0,0,0,0,0)}+\sum_{n, k_{l}, k_{s}, \ell_{l}, \ell_{S}} \hat{\tilde{H}}^{\left(n, k_{1}, \ell_{l}, k_{s}, \ell_{s}\right)}
$$

where the quintuple of integers $\left(n, k_{\mathrm{I}}, \ell_{I}, k_{S}, \ell_{S}\right)$ fulfills the resonance condition defined in Eq. $(10)^{31,32}$. 
In principle, there are infinitely many possible combinations of setting the frequencies $\omega_{\mathrm{r}}, \omega_{\mathrm{m}}^{(\mathrm{I})}$, $\omega_{\mathrm{m}}^{(\mathrm{S})}$ and the two $\mathrm{CW}$ rf-field amplitudes $\omega_{1, \mathrm{CW}}^{(\mathrm{I})}$ and $\omega_{1, \mathrm{CW}}^{(\mathrm{S})}$ to match a heteronuclear dipolar recoupling condition. In the following, we will focus on the resonance condition employed for the ${ }^{\text {RESPIRATION } C P}$ sequence as it was proposed in the original $\operatorname{paper}^{22}$. Here the entire element is rotor synchronized and, therefore,

$$
\omega_{\mathrm{r}}=\omega_{\mathrm{m}}^{(\mathrm{S})}=\omega_{\mathrm{m}}^{(\mathrm{l})}
$$

Additionally, the effective flip angle for the two short pulses were set to be identical with the flip angle varying in the interval $5-175^{\circ}$ for the short-pulse implementation shown in Fig. 1A. This implies that the two CW rf fields in the Floquet description are identical, $\omega_{1, \mathrm{CW}}^{(\mathrm{I})}=\omega_{1, \mathrm{CW}}^{(\mathrm{S})}$ but not cyclic over the time $\tau_{\mathrm{m}}^{(\Lambda)}$.

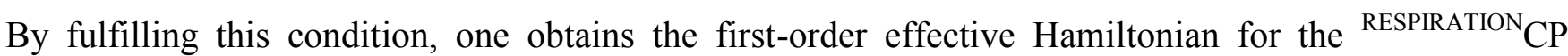
sequence

$$
\hat{\bar{H}}^{(1)}=\sum_{\substack{n_{0}=-2 \\ n_{0} \neq 0}}^{2} \sum_{k_{1}=-\infty}^{\infty} \hat{\tilde{H}}^{\left(n_{0}, k_{\mathrm{I}}, \pm 1,-\left(k_{\mathrm{I}}+n_{0}\right), \mp 1\right)}
$$

where $\ell_{\mathrm{I}}=-\ell_{\mathrm{S}}= \pm 1$ is required for ZQ recoupling.

In Fig. $1 \mathrm{C}$ we show an alternate implementation of the ${ }^{\mathrm{RESPIRATION}} \mathrm{CP}$ experiment where we have replaced the short pulses by $\mathrm{CW}$ irradiation on both channels leading to a phase-alternating recoupling scheme superimposed on the $\mathrm{CW}$ irradiation on the I-spin channel and only $\mathrm{CW}$ irradiation on the S-spin channel. In the following we will call this version $\mathrm{CW}-{ }^{\mathrm{RESPIRATION}} \mathrm{CP}$. This sequence can be described by four characteristic frequencies as shown in Fig. 1D. In this case, it turns out that the description can often be simplified to three frequencies if the two CW rf-field amplitudes have the same magnitude. By applying an additional slow amplitude modulation on either of the involved rf channels (Fig. 1E shows an amplitude modulation on the I channel), an adiabatic passage through the recoupling condition can be achieved similar to the one in adiabatic Hartmann-Hahn $\mathrm{CP}^{33}$ (see Fig. 1F). 
For $\mathrm{CW}-{ }^{\text {RESPIRATION }} \mathrm{CP}$, we have no amplitude-modulation part on the S-channel and in this case $\omega_{k}^{(\mathrm{s})}=0$ for all $k$ and the description simplifies to four frequencies with the Fourier components of the Hamiltonian given by $\hat{\tilde{H}}^{\left(n, k_{1}, \ell_{1}, \ell_{s}\right)}$. By employing the same conditions as proposed for the ${ }^{\text {RESPIRATION }} \mathrm{CP}$ sequence, $\omega_{1, \mathrm{CW}}^{(\mathrm{I})}=\omega_{1, \mathrm{CW}}^{(\mathrm{S})}$ and $\omega_{\mathrm{r}}=\omega_{\mathrm{m}}^{(\mathrm{I})}$, the first-order effective Hamiltonian for ZQ polarization transfer is given by

$$
\hat{\bar{H}}^{(1)}=\hat{\tilde{H}}^{( \pm 2, \mp 2, \pm 1, \mp 1)}+\hat{\tilde{H}}^{( \pm 2, \mp 2, \mp 11, \pm 1)}+\hat{\tilde{H}}^{( \pm 1, \mp 1, \mp 1, \pm 1)}+\hat{\tilde{H}}^{( \pm 1, \mp 1, \pm 1, \mp 1)}
$$

In order to determine the size of the effective heteronuclear dipolar Hamiltonian, the Fourier components according to Eq. (9) have to be calculated. In Fig. 2 the Fourier coefficients are provided as functions of the indices $k_{\mathrm{I}}, k_{\mathrm{S}}$ and the rf-field amplitude $\omega_{1}^{(\Lambda)}$ of either modulated fields in Fig. 1B. Here, we employ the conditions that the rf-field strength of the modulated part is determined according to the original ${ }^{\text {RESPIRATION }} \mathrm{CP}$ sequence in Fig. 1A by requiring $\omega_{1}^{(\mathrm{I})}=\omega_{1}^{(\mathrm{S})}$ under the short pulse and that the amplitude on the I-channel is constant throughout the sequence. Both I- and S-channels are spanning the same time $\tau_{\mathrm{m}}^{(\mathrm{I})}=\tau_{\mathrm{m}}^{(\mathrm{S})}=\tau_{\mathrm{r}}$ with short pulses of duration $\tau_{\mathrm{p}}^{(\mathrm{IS})}=\frac{1}{15} \tau_{\mathrm{m}}^{(\mathrm{IS})}$.

\section{Figure 2}

We can discern some features of the Fourier coefficients $a_{k}^{(\Lambda)}$ for the ${ }^{\text {RESPIRATION }} \mathrm{CP}$ sequence from the plots of their magnitude for the I-channel (Fig. 2A/B) and S-channel (Fig. 2C/D). First of all, the coefficients for the S-channel are concentrated in the real part $a_{k}^{(S)}$ around $k_{\mathrm{S}}=0$ (Fig. 2C/D) for finite but short pulses with $\tau_{\mathrm{p}}^{(\mathrm{IIS})}<<\tau_{\mathrm{r}}$. For the I-channel the size of the coefficients $a_{k}^{(I)}$ have real and imaginary contributions (Fig. 2A/B) with a maximum near $k_{\mathrm{I}} \approx \omega_{1}^{(\mathrm{I})} / \omega_{\mathrm{m}}$. For experimentally realistic rffield amplitudes, the significant contribution to the effective Hamiltonian is mainly given by the values $k_{I}, k_{S} \in[-10 ; 10]$. The Fourier coefficients for the $\mathrm{CW}-{ }^{\mathrm{RESPIRATION}} \mathrm{CP}$ are the same as for the XiX scheme and can be found in the literature ${ }^{30}$. 


\section{Data and Discussions}

Combining the Fourier coefficients $a_{k_{\Lambda}}^{(\Lambda)}$ (Fig. 2) with the analytical first-order effective Hamiltonian (Eqs. (13) and (14)) and the Fourier components of the Hamiltonian (Eq. (9)) allows us to calculate the strength of the effective heteronuclear dipolar-coupling Hamiltonian for both ${ }^{\text {RESPIRATION }} \mathrm{CP}$ and $\mathrm{CW}-{ }^{\text {RESPIRATION }} \mathrm{CP}$. We use this analytical first-order effective Hamiltonian to calculate the $\hat{I}_{x} \rightarrow \hat{S}_{x}$ coherence transfer efficiency as function of the rf-field amplitude and mixing time at a MAS frequency of $16.7 \mathrm{kHz}$ and a pulse length of $\tau_{\mathrm{p}}^{(\mathrm{I} / \mathrm{S})}=4 \mu \mathrm{s}$ for ${ }^{\text {RESPIRATION }} \mathrm{CP}$ (Fig. 3A) and the corresponding $\mathrm{CW}$ rf-field amplitude with the same flip angle for $\mathrm{CW}-{ }^{\mathrm{RESPIRATION}} \mathrm{CP}$ (Fig. $3 \mathrm{~B}$ ). The rf-field amplitudes are changed by setting $\omega_{1}=\omega_{1}^{(\mathrm{I})}=\omega_{1}^{(\mathrm{S})}$ with constant field strength over the entire element for ${ }^{\text {RESPIRATION }} \mathrm{CP}$.

The two plots of Fig. 3A/B are almost identical, indicating that the effective first-order Hamiltonians for ${ }^{\text {RESPIRATION }}$ CP (Fig. 3A) and CW- ${ }^{\text {RESPIRATION }}$ CP (Fig. 3B) are almost identical under these conditions. By simultaneously changing the rf-field amplitude on both channels we make sure that the resonance conditions are unchanged and $\omega_{1, \mathrm{CW}}^{(\mathrm{I})}=\omega_{1, \mathrm{CW}}^{(\mathrm{S})}$ is always fulfilled. One can see that the effective dipolar scaling factor is small at low rf-field amplitudes (slow polarization transfer) but in the range $\omega_{1, \mathrm{~m}}^{(\Lambda)} \in\left[0.75 \omega_{\mathrm{r}} ; 2 \omega_{r}\right]$ the scaling factor is only changing slightly as the rate of polarization transfer is almost constant. For ${ }^{\text {RESPIRATION }} \mathrm{CP}$, this feature can be understood from the magnitude of the Fourier coefficients (Fig. 2A and 2C) shown combined with Eq. (13) which shows that only terms fulfilling the condition $k_{s}=-k_{\mathrm{I}}-n_{0}$ will contribute to the effective first-order dipolar coupling Hamiltonian. For low rf-field strength $\left(\omega_{1} / \omega_{\mathrm{r}}<0.75\right)$ the Fourier coefficients are largest for $k_{I}=k_{S}=0$ which cannot contribute to the Hamiltonian since $n_{0}=0$ does not exist. For higher rf-field amplitudes, the maximum of the Fourier coefficients shifts to $k_{I}= \pm 1, \pm 2$ for the I spin while $k_{S}=0$ remains the maximum for the $\mathrm{S}$ spin. In general, the maximum transfer efficiency depends on the relative magnitude of the $n_{0}= \pm 1$ 
and $n_{0}= \pm 2$ components of the Fourier coefficients of the dipolar coupling $\left(\omega_{\mathrm{IS}}^{\left(n_{0}\right)}\right)$ in a similar fashion as shown in the case of $\mathrm{XiX}^{\mathrm{CW}} \operatorname{DREAM}^{34}$. Thus, largest Fourier coefficients $a_{k_{\mathrm{I}}}^{(\mathrm{I})}$ are desired for $k_{I}= \pm 1 ; \pm 2$ to maximize the scaling factor. For $\mathrm{CW}-{ }^{\mathrm{RESPIRATION}} \mathrm{CP}$ similar requirements are found.

In Fig. 3C and 3D, experimental data are presented for ${ }^{15} \mathrm{~N}-{ }^{13} \mathrm{C}_{\alpha}$ coherence transfer for a powder sample of uniformly ${ }^{13} \mathrm{C},{ }^{15} \mathrm{~N}$-labeled alanine by using either ${ }^{\mathrm{RESPIRATION}} \mathrm{CP}$ (Fig. $3 \mathrm{C}$ ) or $\mathrm{CW}$ ${ }^{\text {RESPIRATION }} \mathrm{CP}$ (Fig. 3D) as a mixing element. The data were recorded on a $14.4 \mathrm{~T}$ spectrometer (600 MHz ${ }^{1} \mathrm{H}$ Larmor frequency) at a MAS frequency of $16.7 \mathrm{kHz}$ and by applying rf irradiation onresonance for ${ }^{15} \mathrm{~N}$ and ${ }^{13} \mathrm{C}_{\alpha}$ nuclei. The same parameters for the mixing elements were used as for the simulations shown in Fig. 3A and 3B. Overall, the experimental performance is comparable to the theoretical predictions. However, the maximum experimental transfer efficiency is around $40 \%$ compared to $75 \%$ for the theoretical calculated. This difference may be explained by a number of effects. In particular the lack of CSA contributions in the theoretical calculation which can contribute through higher-order cross terms. The importance of CSA terms can be seen from experimental data recorded on a $850 \mathrm{MHz}$ spectrometer with all other settings being identical (see supporting material Fig. S1 C and D) which shows significantly lower transfer efficiencies. Other effects are insufficient ${ }^{1} \mathrm{H}$ decoupling during the mixing element where we have experimentally used $100 \mathrm{kHz} \mathrm{CW}$ irradiation. In general higher-order terms and rf-field inhomogeneities leading to mismatch are not present in the calculation. All these effects will decrease the transfer efficiency.

\section{Figure 3}

The overall theoretical and experimental performance for the two experiments as shown in Fig. 3 is indeed very similar under the typical conditions used in ${ }^{\text {RESPIRATION }} \mathrm{CP}$. However, by choosing a condition where the short pulses correspond to $\pi$-pulses $\left(\omega_{1, \mathrm{CW}}^{(\mathrm{I})}=\omega_{1, \mathrm{CW}}^{(\mathrm{S})}=\frac{1}{2} \omega_{\mathrm{r}}\right)$ for both ${ }^{\mathrm{RESPIRATION}} \mathrm{CP}$ (keeping $\omega_{\mathrm{r}}=\omega_{\mathrm{m}}^{(\mathrm{S})}=\omega_{\mathrm{m}}^{(\mathrm{l})}$ ) and $\mathrm{CW}-{ }^{\text {RESPIRATION }} \mathrm{CP} \quad$ (keeping $\omega_{\mathrm{r}}=\omega_{\mathrm{m}}^{(\mathrm{l})}$ ), the effective first-order Hamiltonians will indeed be different for the two experiments. Under these conditions additional 
resonance terms have to be considered in Eq. (10) since in addition the double-quantum resonance conditions $\omega_{1, \mathrm{CW}}^{(\mathrm{I})}+\omega_{1, \mathrm{CW}}^{(\mathrm{S})}=\omega_{\mathrm{r}}$ is fulfilled. Simultaneous matching of the zero-quantum and doublequantum resonance conditions is often detrimental for efficient polarization transfer. In addition to the heteronuclear recoupling conditions one will also fulfill the homonuclear dipolar recoupling condition $(\text { HORROR })^{35}$ under these settings for both ${ }^{\text {RESPIRATION }} \mathrm{CP}$ and $\mathrm{CW}-{ }^{\text {RESPIRATION }}{ }^{\mathrm{CP}}$. A general description of the homonuclear recoupling condition has been shown for the recently described $\mathrm{XiX}^{\mathrm{CW}}$ DREAM experiment $^{34}$. These homonuclear resonance conditions should also be avoided in both ${ }^{\text {RESPIRATION } C P}$ variants. Hence, when the two short pulses in ${ }^{\operatorname{RESPIRATION}} \mathrm{CP}$ are set to be identical with the effective flip angle between $\omega_{1}^{(\Lambda)} \tau_{\mathrm{p}}^{(\Lambda)}=5^{\circ}$ and $\omega_{1}^{(\Lambda)} \tau_{\mathrm{p}}^{(\Lambda)}=175^{\circ}$, the two sequences give similar experimental and theoretical results and differences between the two implementations should be due to different secondorder contributions. We are currently working on a full second-order description to analyze these differences in more detail.

The CW components of the rf field in ${ }^{\text {RESPIRATION }} \mathrm{CP}$ or $\mathrm{CW}-{ }^{\mathrm{RESPIRATION}} \mathrm{CP}$ sequences are part of the resonance conditions but they do not provide the recoupling as they do in the original $\mathrm{CP}$ experiment. The rf-field inhomogeneities in standard Hartmann-Hahn CP translate directly into a mismatch of the resonance conditions, i.e., the modified Hartmann-Hahn conditions under MAS ( $\left.\omega_{1, \mathrm{CW}}^{(\mathrm{I})} \pm \omega_{1, \mathrm{CW}}^{(\mathrm{S})}=n_{0} \omega_{\mathrm{r}}\right)$. For the ${ }^{\text {RESPIRATION }} \mathrm{CP}$ variants, they mainly achieve a spin-lock of the polarization in the rotating frame. By fulfilling that $\omega_{1, \mathrm{CW}}^{(\mathrm{I})}=\omega_{1, \mathrm{CW}}^{(\mathrm{S})}$, the resonance conditions are unchanged regarding a scaling of the rf-field amplitude with the same amount on both channels. This make the performance of the sequence more stable towards variations of the effective rf field strengths over a large sample volume. The actual recoupling for ${ }^{\mathrm{RESPIRATION}} \mathrm{CP}$ variants are achieved by the phase-alternating component of the time-dependent rf field which has a modulation frequency matched to the spinning frequency. Here, the modulation frequency determines the resonance condition and the rf-field amplitude will only indirectly change the Fourier coefficients that determine the strength of the effective dipolar coupling. This slow change in the effective dipolar coupling strength can be seen from Fig. 3 
where the rate of polarization transfer is almost constant in the range $\omega_{1, m}^{(\Lambda)} \in\left[0.75 \omega_{r} ; 2 \omega_{r}\right]$. As an added benefit, the $n_{0}=1$ and the $n_{0}=2$ resonance conditions can be matched simultaneously leading to a different angle dependence than for the standard $\gamma$-encoded Hartmann-Hahn CP experiment. This results in a theoretical maximum of the polarization transfer of $75 \%$ compared to $73 \%$ for $\gamma$-encoded sequences $^{22,35}$.

In general, the CP efficiency can be improved by adiabatically sweeping the rf field strength through the resonance condition which leads to higher transfer and also partially compensates the rffield inhomogeneity. From the $\mathrm{CW}$ implementation of the ${ }^{\mathrm{RESPIRATION}} \mathrm{CP}$ experiment, it is easy to see that the sequence can be modified to obtain an adiabatic polarization transfer by applying an additional amplitude sweep on top of either the $\omega_{1, \mathrm{CW}}^{(\mathrm{I})}$ or $\omega_{1, \mathrm{CW}}^{(\mathrm{S})}$ rf fields. This is illustrated in Fig. 1E/F where the sweep is applied on the I-channel. The same is true for the original sequence ${ }^{\text {RESPIRATION }}{ }^{\mathrm{CP}}$ as shown in the adiabatic ${ }^{\text {RESPIRATION }} \mathrm{CP}$ experiment ${ }^{23}$. We have compared the ${ }^{1} \mathrm{H}^{-15} \mathrm{~N}$ coherence transfer by employing either adiabatic $\mathrm{CP}$ or adiabatic $\mathrm{CW}-{ }^{\mathrm{RESPIRATION}} \mathrm{CP}$ to mediate the transfer. Figure $4 \mathrm{~A}$ shows a proton-detected 2D ${ }^{15} \mathrm{~N}-{ }^{1} \mathrm{H}$ correlation spectrum of for uniformly ${ }^{2} \mathrm{H},{ }^{13} \mathrm{C},{ }^{15} \mathrm{~N}$-labeled ubiquitin with $100 \%$ back-exchanged amide protons. The data was recorded at a spinning frequency of $90.9 \mathrm{kHz}$ on a $850 \mathrm{MHz}$ spectrometer using adiabatic $\mathrm{CW}_{-}{ }^{\mathrm{RESPIRATION}} \mathrm{CP}$ as the two mixing elements for both initial ${ }^{1} \mathrm{H}-{ }^{15} \mathrm{~N}$ transfer and ${ }^{15} \mathrm{~N}-{ }^{1} \mathrm{H}$ transfer after a free evolution period $\mathrm{t}_{1}$. The signal intensities obtained using adiabatic $\mathrm{CP}$ and adiabatic $\mathrm{CW}-{ }^{\mathrm{RESPIRATION}} \mathrm{CP}$ are compared in Fig. 4B for individual well-resolved peaks. For adiabatic CP we used a mixing time of $1.00 \mathrm{~ms}$ and $\mathrm{rf}$ field strengths with mean of $70.7 \mathrm{kHz}$ (and a tangent shape with $\frac{\Delta}{2 \pi}=14.1 \mathrm{kHz}$ and $\frac{d_{\text {est }}}{2 \pi}=2.49 \mathrm{kHz}$ ) on ${ }^{1} \mathrm{H}$ channel and $16.4 \mathrm{kHz}$ on ${ }^{15} \mathrm{~N}$. For adiabatic CW- ${ }^{\text {RESPIRATION }} \mathrm{CP}$ the mixing time was $1.868 \mathrm{~ms}$ and $\mathrm{rf}$ field strengths of $\omega_{1, m}=100 \mathrm{kHz}$ and $\omega_{1, \mathrm{CW}}=20 \mathrm{kHz}$ (and a tangent shape with $\frac{\Delta}{2 \pi}=12.0 \mathrm{kHz}$ and $\frac{d_{e s t}}{2 \pi}=2.6 \mathrm{kHz}$ ) on ${ }^{1} \mathrm{H}$ channel and 20 $\mathrm{kHz}$ on ${ }^{15} \mathrm{~N}$. These conditions were employed for both initial ${ }^{1} \mathrm{H}-{ }^{15} \mathrm{~N}$ transfer and ${ }^{15} \mathrm{~N}-{ }^{1} \mathrm{H}$ transfer for both experiments. Additional experimental parameters are given in Table S1 of the Supporting Material. 


\section{Figure 4}

From the data in Fig. 4B we see that adiabatic-CW- ${ }^{\text {RESPIRATION }} \mathrm{CP}$ performs slightly better or similar than adiabatic $\mathrm{CP}$ with individual peak gains of up to $15 \%$ in the recorded $2 \mathrm{D}$ spectra after both initial ${ }^{1} \mathrm{H}-{ }^{15} \mathrm{~N}$ transfer and ${ }^{15} \mathrm{~N}-{ }^{1} \mathrm{H}$ transfer. Thus, we see a gain of around $7 \%$ for the individually transfer steps.

\section{Conclusions}

In conclusion, we have presented a five-mode Floquet treatment to analyze ${ }^{\text {RESPIRATION }}{ }^{\mathrm{CP}}$. We have shown that ${ }^{\mathrm{RESPIRATION}} \mathrm{CP}$ or $\mathrm{CW}-{ }^{\mathrm{RESPIRATION}} \mathrm{CP}$ theoretically can be separated into a timeindependent $\mathrm{CW}$ part and a time-dependent component with zero net flip angle. Based on this description, we have shown that the first-order effective Hamiltonians is almost identical by using the original ${ }^{\text {RESPIRATION }} \mathrm{CP}$ scheme compared to the $\mathrm{CW}-{ }^{\mathrm{RESPIRATION}} \mathrm{CP}$ scheme for polarization transfer between spin- $1 / 2$ nuclei when the short interleaved pulses have identical flip-angle varying in the interval $5-175^{\circ}$. In general, the proposed description opens up for the possibility to understand finite pulse effects of the short pulses and to analyze higher-order terms which will be affected differently for the two schemes.

\section{Acknowledgment}

We acknowledge support from the Danish National Research Foundation (DFF-4090-00223; DNRF59). A.B.N. acknowledges an ETH fellows postdoctoral grant. This work was supported by the Swiss National Science Foundation (Grants 200020_146757 and 200020_159707) 


\section{References}

1. Wasmer, C., Lange, A., Van Melckebeke, H., Siemer, A.B., Riek, R. \& Meier, B.H. Amyloid fibrils of the HET-s(218-289) prion form a beta solenoid with a triangular hydrophobic core. Science 319, 1523-1526 (2008).

2. Schütz, A.K., Vagt, T., Huber, M., Ovchinnikova, O.Y., Cadalbert, R., Wall, J., Güntert, P., Böckmann, A., Glockshuber, R. \& Meier, B.H. Atomic-Resolution Three-Dimensional Structure of Amyloid $\beta$ Fibrils Bearing the Osaka Mutation. Angewandte Chemie International Edition 54, 331-335 (2015).

3. Loquet, A., Sgourakis, N.G., Gupta, R., Giller, K., Riedel, D., Goosmann, C., Griesinger, C., Kolbe, M., Baker, D., Becker, S. \& Lamge, A. Atomic model of the type III secretion system needle. Nature 486, 276-279 (2012).

4. Castellani, F., van Rossum, B., Diehl, A., Schubert, M., Rehbein, K. \& Oschkinat, H.. Structure of a protein determined by solid-state magic-angle-spinning NMR spectroscopy. Nature 420, 98102 (2002).

5. Lewandowski, J.R., Halse, M.E., Blackledge, M. \& Emsley, L. Direct observation of hierarchical protein dynamics. Science 348, 578-581 (2015).

6. Knight, M.J., Pell, A.J., Bertini, I., Felli, I.C., Gonnelli, L., Pierattelli, R., Herrmann, T., Emsley, L. \& Pintacuda, G. Structure and backbone dynamics of a microcrystalline metalloprotein by solid-state NMR. Proceedings of the National Academy of Sciences 109, 11095-11100 (2012).

7. Ladizhansky, V. Homonuclear dipolar recoupling techniques for structure determination in uniformly 13C-labeled proteins. Solid State Nucl Magn Reson 36, 119-128 (2009).

8. Griffin, R.G. Dipolar recoupling in MAS spectra of biological solids. Nature structural biology 5, 508-512 (1998).

9. Nielsen, N.C., Strassø, L.A. \& Nielsen, A.B. Dipolar Recoupling. Topics in Current Chemistry 306, 1-47 (2012).

10. Meier, B.H. Polarization Transfer and Spin Diffusion in Solid-State NMR. Advances in Magnetic and Optical Resonance 18, 1-116 (1994).

11. Hartmann, S.R. \& Hahn, E.L. Nuclear Double Resonance in Rotating Frame. Phys Rev 128, 2042 (1962).

12. Stejskal, E.O., Schaefer, J. \& Waugh, J.S. Magic-Angle Spinning and Polarization Transfer in Proton-Enhanced Nmr. J Magn Reson 28, 105-112 (1977).

13. Schaefer, J., Mckay, R.A. \& Stejskal, E.O. Double-Cross-Polarization Nmr of Solids. J Magn Reson 34, 443-447 (1979).

14. Schuetz, A., Wasmer, C., Habenstein, B., Verel, R., Greenwald, J., Riek, R., Böckmann, A. \& Meier, B.H. Protocols for the Sequential Solid-State NMR Spectroscopic Assignment of a Uniformly Labeled 25 kDa Protein: HET-s(1-227). ChemBioChem 11, 1543-1551 (2010).

15. Habenstein, B., Wasmer, C., Bousset, L., Sourigues, Y., Schütz, A., Loquet, A., Meier, B.H., Melki, R. \& Böckmann, A. Extensive de novo solid-state NMR assignments of the $33 \mathrm{kDa}$ Cterminal domain of the Ure2 prion. J Biomol NMR 51, 235-243 (2011).

16. Khaneja, N., Kehlet, C., Glaser, S.J. \& Nielsen, N.C. Composite dipolar recoupling: anisotropy compensated coherence transfer in solid-state nuclear magnetic resonance. $J$ Chem Phys 124, 114503 (2006).

17. Hediger, S., Meier, B.H. \& Ernst, R.R. Cross-Polarization under Fast Magic-Angle SampleSpinning Using Amplitude-Modulated Spin-Lock Sequences. Chemical Physics Letters 213, 627-635 (1993).

18. Kolbert, A.C. \& Bielecki, A. Broadband Hartmann-Hahn Matching in Magic-Angle Spinning NMR via an Adiabatic Frequency Sweep. Journal of Magnetic Resonance, Series A 116, 29-35 (1995).

19. Lin, J., Griffin, R.G. \& Khaneja, N. Recoupling in solid state NMR using $\gamma$ prepared states and phase matching. J Magn Reson 212, 402-411 (2011). 
20. Bjerring, M. \& Nielsen, N.C. Solid-state NMR heteronuclear coherence transfer using phase and amplitude modulated rf irradiation at the Hartmann-Hahn sideband conditions. Chemical Physics Letters 382, 671-678 (2003).

21. Wei, D., Akbey, U., Paaske, B., Oschkinat, H., Reif, B., Bjerring, M. \& Nielsen, N.C. Optimal 2H rf Pulses and 2H-13C Cross-Polarization Methods for Solid-State 2H MAS NMR of Perdeuterated Proteins. The Journal of Physical Chemistry Letters 2, 1289-1294 (2011)

22. Jain, S., Bjerring, M. \& Nielsen, N.C. Efficient and Robust Heteronuclear Cross-Polarization for High-Speed-Spinning Biological Solid-State NMR Spectroscopy. The Journal of Physical Chemistry Letters 3, 703-708 (2012).

23. Nielsen, A.B., Jain, S., Ernst, M., Meier, B.H. \& Nielsen, N.C. Adiabatic Rotor-Echo-ShortPulse-Irradiation mediated cross-polarization. J Magn Reson 237, 147-151 (2013).

24. Jain, S.K., Nielsen, A.B., Hiller, M., Handel, L., Ernst, M., Oschkinat, H., Akbey, U. \& Nielsen, N.C. Low-power polarization transfer between deuterons and spin-1/2 nuclei using adiabatic RESPIRATIONCP in solid-state NMR. Physical Chemistry Chemical Physics 16, 2827-2830 (2014).

25. Basse, K., Jain, S.K., Bakharev, O. \& Nielsen, N.C. Efficient polarization transfer between spin1/2 and N nuclei in solid-state MAS NMR spectroscopy. J Magn Reson 244, 85-89 (2014).

26. Mehring, M. Principles of High Resolution NMR in Solids. (Springer-Verlag, New York; 1983).

27. Vinogradov, E., Madhu, P.K. \& Vega, S. A bimodal Floquet analysis of phase modulated LeeGoldburg high resolution proton magic angle spinning NMR experiments. Chemical Physics Letters 329, 207-214 (2000).

28. Weintraub, O. \& Vega, S. Floquet Density Matrices and Effective Hamiltonians in MagicAngle-Spinning NMR Spectroscopy. Journal of Magnetic Resonance, Series A 105, 245-267 (1993).

29. Shirley, J.H. Solution of the Schrödinger Equation with a Hamiltonian Periodic in Time. Phys Rev 138, 979-987 (1965).

30. Ernst, M., Geen, H. \& Meier, B.H. Amplitude-modulated decoupling in rotating solids: A bimodal Floquet approach. Solid State Nucl Mag 29, 2-21 (2006).

31. Scholz, I., van Beek, J.D. \& Ernst, M. Operator-based Floquet theory in solid-state NMR. Solid State Nucl Mag 37, 39-59 (2010).

32. Leskes, M., Madhu, P.K. \& Vega, S. Floquet theory in solid-state nuclear magnetic resonance. Progress in Nuclear Magnetic Resonance Spectroscopy 57, 345-380 (2010).

33. Hediger, S., Meier, B.H. \& Ernst, R.R. Adiabatic passage Hartmann-Hahn cross polarization in NMR under magic angle sample spinning. Chemical Physics Letters 240, 449-456 (1995).

34. Tan, K.O., Nielsen, A.B., Meier, B.H. \& Ernst, M. Broad-Band DREAM Recoupling Sequence. The Journal of Physical Chemistry Letters 5, 3366-3372 (2014).

35. Nielsen, N.C., Bildsoe, H., Jakobsen, H.J. \& Levitt, M.H. Double-Quantum Homonuclear Rotary Resonance - Efficient Dipolar Recovery in Magic-Angle-Spinning Nuclear-MagneticResonance. J Chem Phys 101, 1805-1812 (1994). 
Figure Graphical Abstract

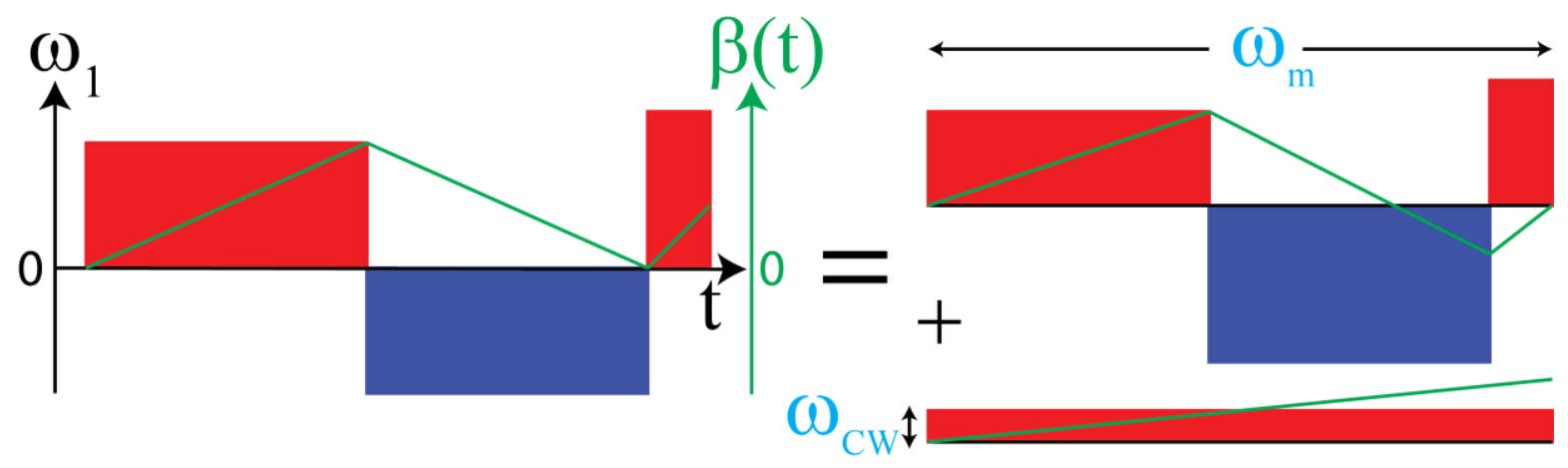


Figure 1
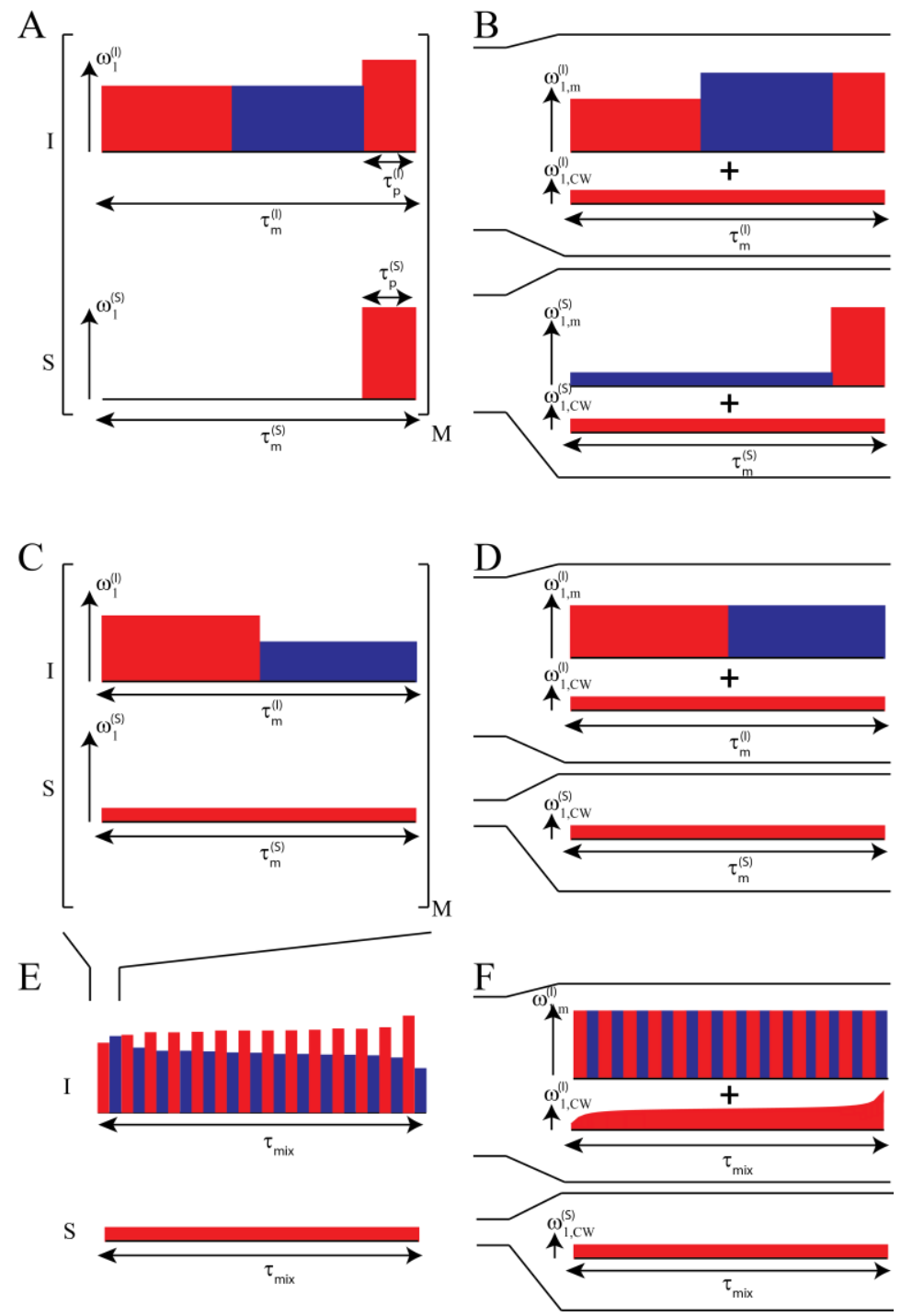

Fig. 1. A representation of ${ }^{\text {RESPIRATION }} \mathrm{CP}(\mathrm{A})$ and $\mathrm{CW}-{ }^{\mathrm{RESPIRATION}} \mathrm{CP}$-element $(\mathrm{C})$ which is repeated $\mathrm{M}$ times. The ${ }^{\text {RESPIRATION }} \mathrm{CP}$ element consists of two phase-alternated pulses on the I-spin rf channel. These are followed by short rf pulses of duration $\tau_{p}$ on the S- and I-spin channels. For the CW- ${ }^{\text {RESPIRATION }}{ }^{\text {CP- }}$ element there is CW rf field on S-channel and a phase- and amplitude-modulated rf field on I-channel. The sequences can be split into a sum of a CW and a phase-modulated rf field (B and D). A schematic representation of the I-spin mixing sequence for adiabatic $\mathrm{CW}$ - ${ }^{\mathrm{RESPIRATION}} \mathrm{CP}$ with mixing time $\tau_{m i x}$ is presented (E) which is split into $\mathrm{CW}$ and a phase-modulated $\mathrm{rf}$ field (F). Red and blue colors indicate $x$ and $-x$ phase of the pulses, respectively. 
Figure 2
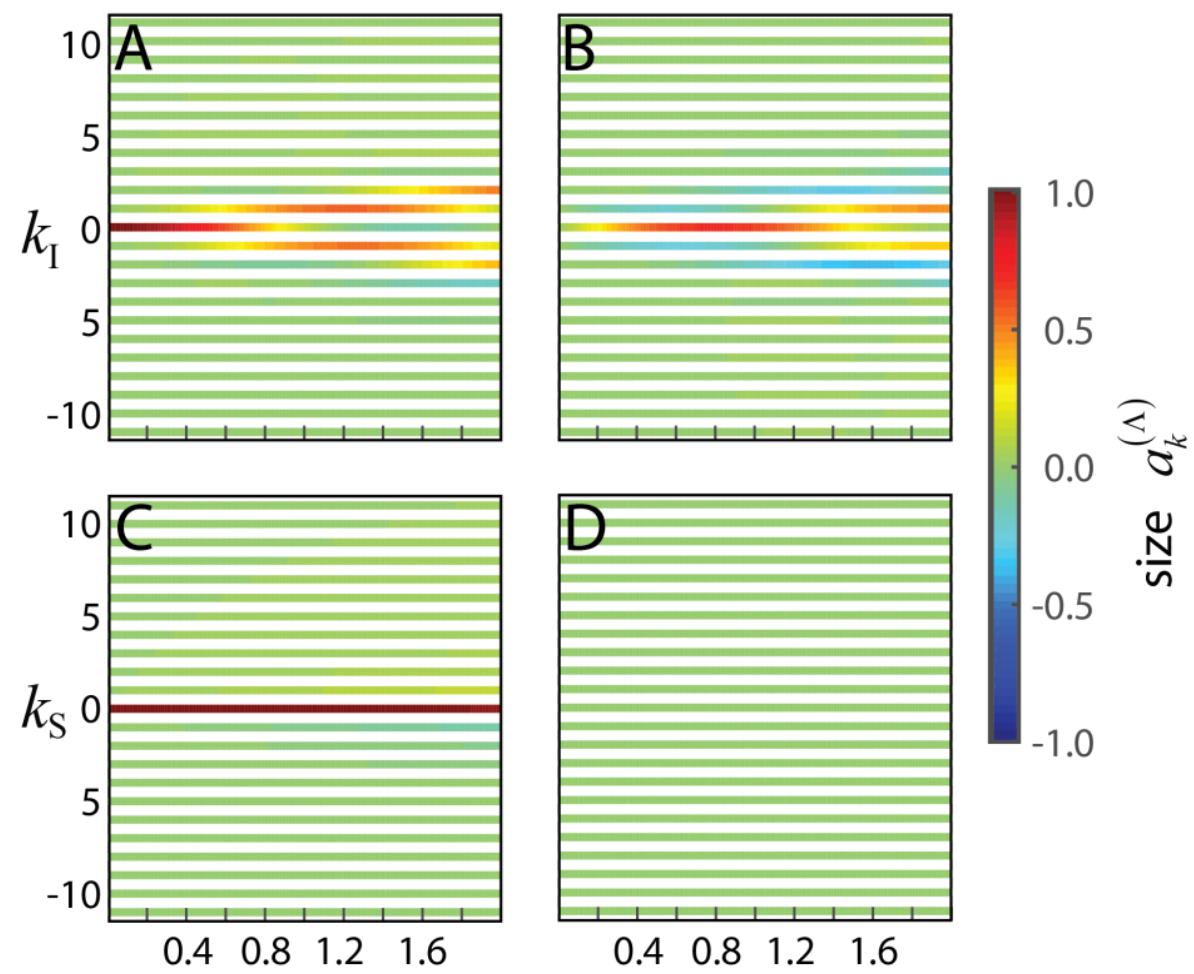

$$
\omega_{1}^{(\Lambda)} / \omega_{\mathrm{r}}
$$

$\omega_{1}^{(\Lambda)} / \omega_{\mathrm{r}}$

Fig. 2. Plot of the magnitude of the Fourier coefficients $a_{k}^{(\Lambda)}$ as function of the indexes $k_{\mathrm{I}}$ and $k_{\mathrm{S}}$, respectively, and the rf field amplitude of the modulated fields. The cycle time is $\tau_{\mathrm{m}}^{(\mathrm{I})}=\tau_{\mathrm{m}}^{(\mathrm{S})}=\tau_{\mathrm{r}}$ and the short pulse time is chosen to $\tau_{\mathrm{p}}^{(\mathrm{I} / \mathrm{S})}=\frac{4}{60} \tau_{\mathrm{m}}^{(\mathrm{I} / \mathrm{S})}$. The same $\mathrm{rf}$ field strengths for all the pulses in the ${ }^{\text {RESPIRATION }} \mathrm{CP}$ sequence in Fig. 1A is employed with $\omega_{1}^{(\mathrm{I})}=\omega_{1}^{(\mathrm{S})}$ to determine the rf amplitude of the modulated fields in Fig. 1B. (A) real part and (B) imaginary part for $\Lambda=\mathrm{I}$ and (C) real part and (D) imaginary part for $\Lambda=\mathrm{S}$. 
Figure 3

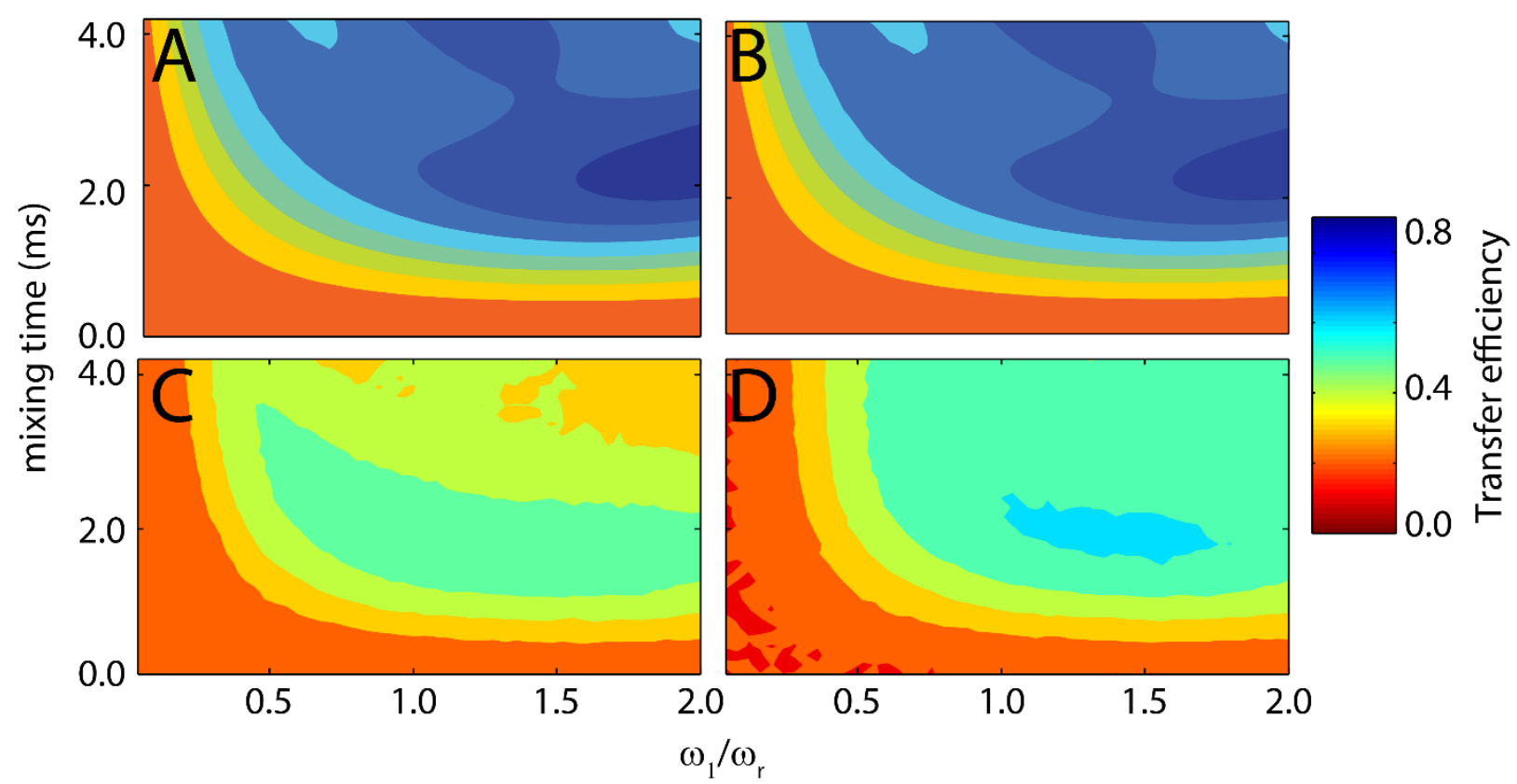

Fig. 3. Analytical (A and B) and experimental (C and D) ${ }^{15} \mathrm{~N} \rightarrow{ }^{13} \mathrm{C}_{\alpha}$ coherence transfer efficiencies (normalized such $100 \%$ designates that all initial magnetization is transferred) for ${ }^{\text {RESPIRATION }}{ }^{\mathrm{CP}}$ (A and C) and $\mathrm{CW}_{-}{ }^{\mathrm{RESPIRATION}} \mathrm{CP}(\mathrm{B}$ and $\mathrm{D})$ as function of $\mathrm{rf}$ field strength (x-axis) and mixing time (y-axis). The time for the repeated elements were $\tau_{\mathrm{m}}^{(\mathrm{I})}=\tau_{\mathrm{m}}^{(\mathrm{S})}=\tau_{\mathrm{r}}=60 \mu \mathrm{s}$ and for ${ }^{\text {RESPIRATION }} \mathrm{CP}$ the short pulse time was $\tau_{\mathrm{p}}^{(\mathrm{I})}=\tau_{\mathrm{p}}^{(\mathrm{s})}=4 \mu \mathrm{s}$. A constant rf field strengths for all the pulses in ${ }^{\text {RESPIRATION }}{ }^{\mathrm{CP}}$ sequence were employed with $\omega_{1}^{(\mathrm{I})}=\omega_{1}^{(\mathrm{S})}=\omega_{1}$ (A and C). For CW- ${ }^{\mathrm{RESPIRATION}} \mathrm{CP}$ (B and D) the rf field strengths of the CW fields are given by $\omega_{1, C W}^{(\mathrm{I})}=\omega_{1, C W}^{(\mathrm{S})}=\omega_{1} \frac{\tau_{p}^{(I / S)}}{\tau_{r}}$ and the modulating part by $\omega_{1, m}^{(\mathrm{I})}=\omega_{1}\left(1 \pm \frac{\tau_{p}^{(I)}}{\tau_{r}}\right)$. Data (C and D) were obtained for uniformly ${ }^{13} \mathrm{C},{ }^{15} \mathrm{~N}$-labeled alanine on a $600 \mathrm{MHz}$ spectrometer at $16.7 \mathrm{kHz}$ spinning. 
Figure 4
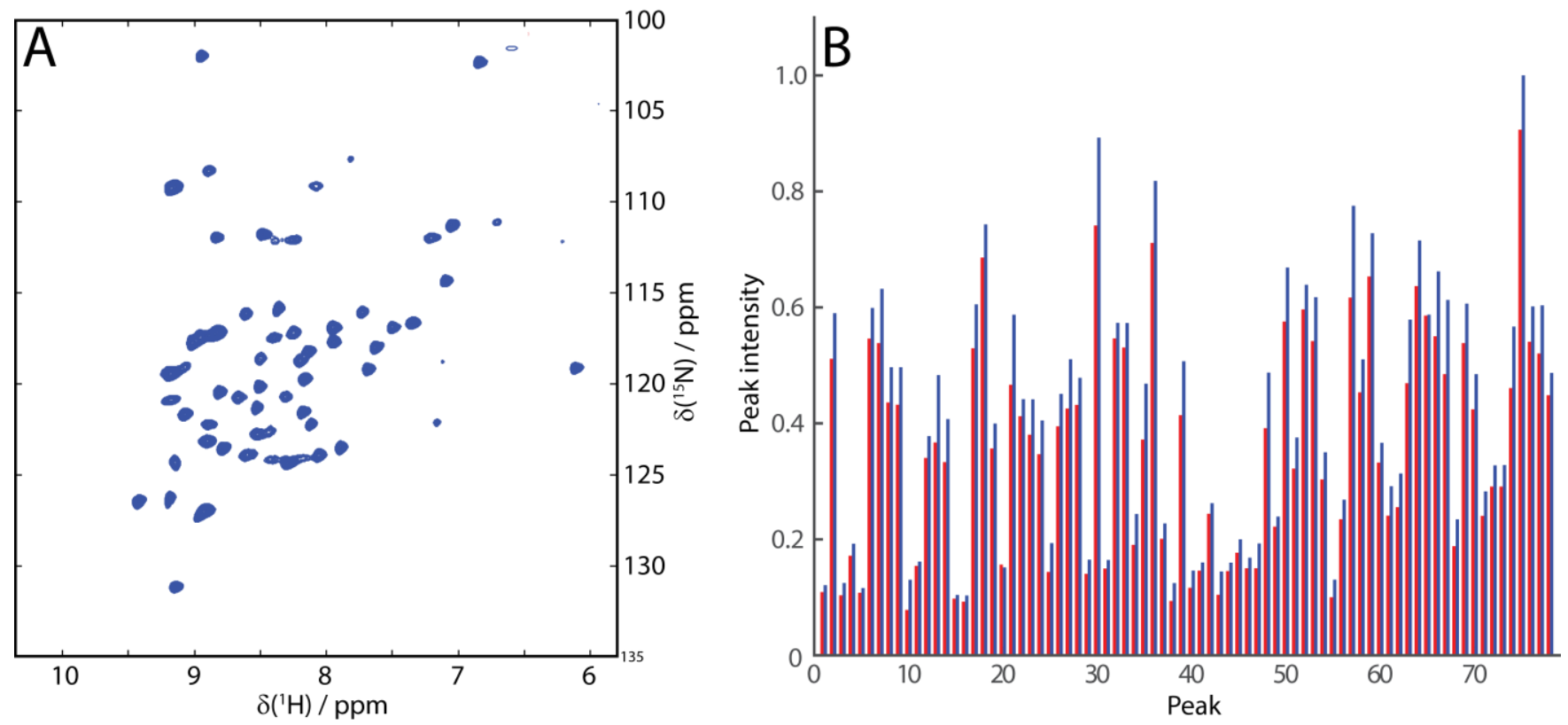

Fig. 4. Experimental 2D spectrum for uniformly ${ }^{2} \mathrm{H},{ }^{13} \mathrm{C},{ }^{15} \mathrm{~N}$-labeled and $100 \%$ back-exchanged ubiquitin recorded on a $850 \mathrm{MHz}$ spectrometer at $90.9 \mathrm{kHz}$ MAS using the adiabatic $\mathrm{CW}-{ }^{\text {RESPIRATION }}{ }^{\mathrm{CP}}$ sequence for ${ }^{1} \mathrm{H}_{-}{ }^{15} \mathrm{~N}$ and ${ }^{15} \mathrm{~N}-{ }^{1} \mathrm{H}$ polarization transfer (A). A comparison for peak intensities (normalized to highest peak intensity) from 2D spectra using adiabatic CP (red) and adiabatic CW- ${ }^{\text {RESPIRATION }}{ }^{\mathrm{CP}}$ (blue) as mixing elements (B). All experimental parameters are given in the supporting material Tables S1. 


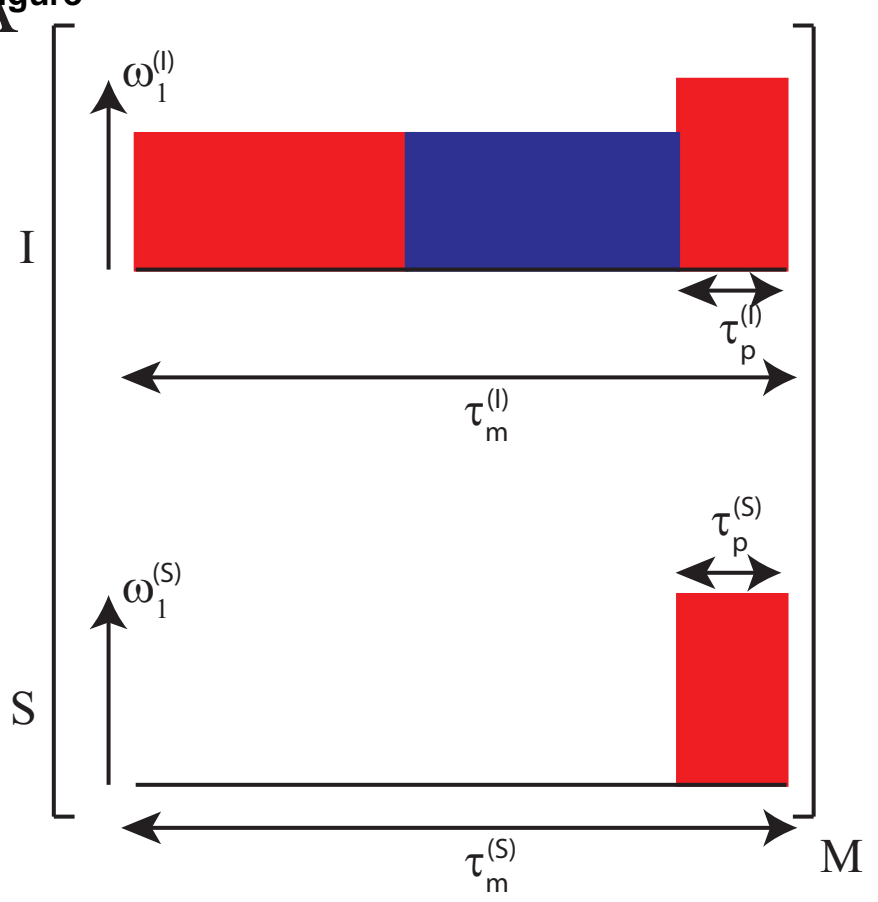

B

C
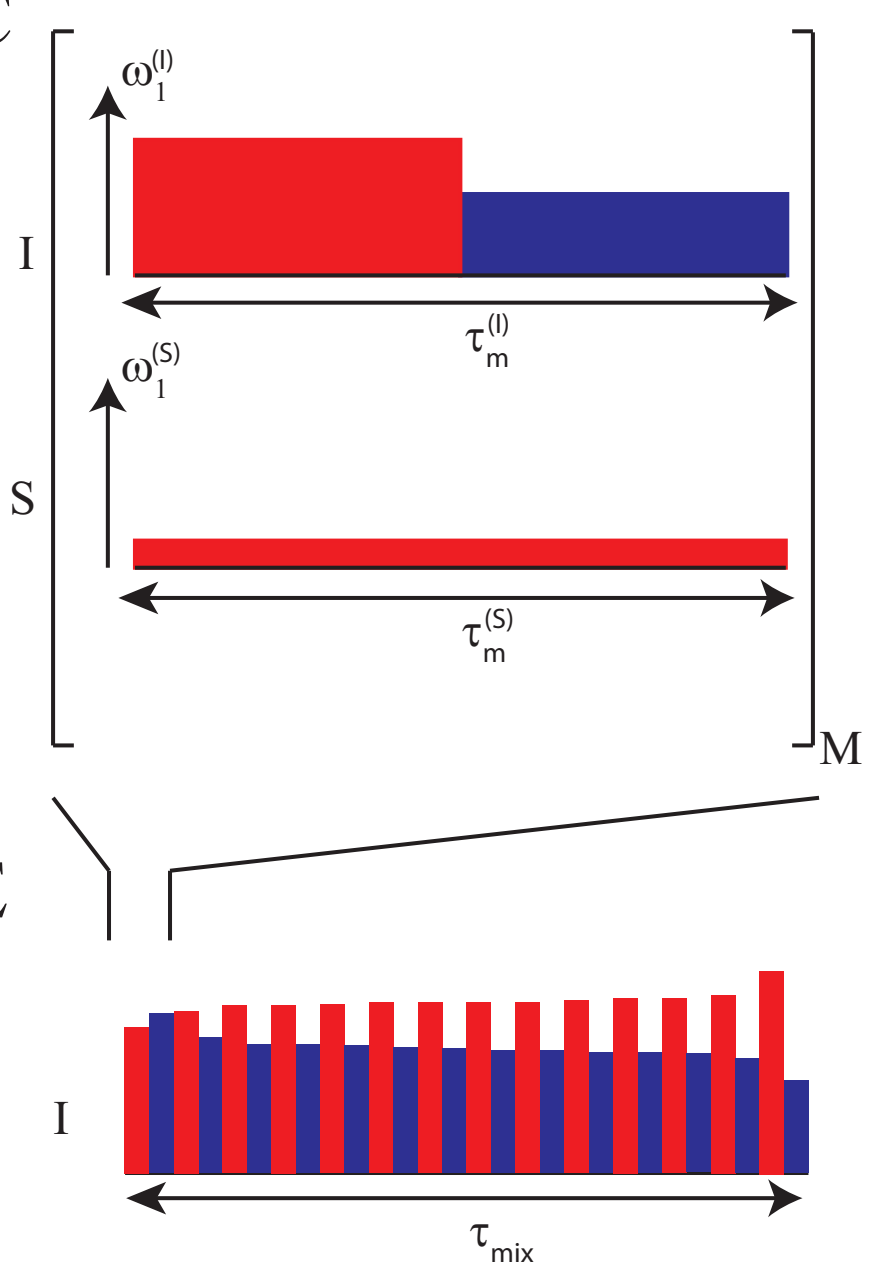

D
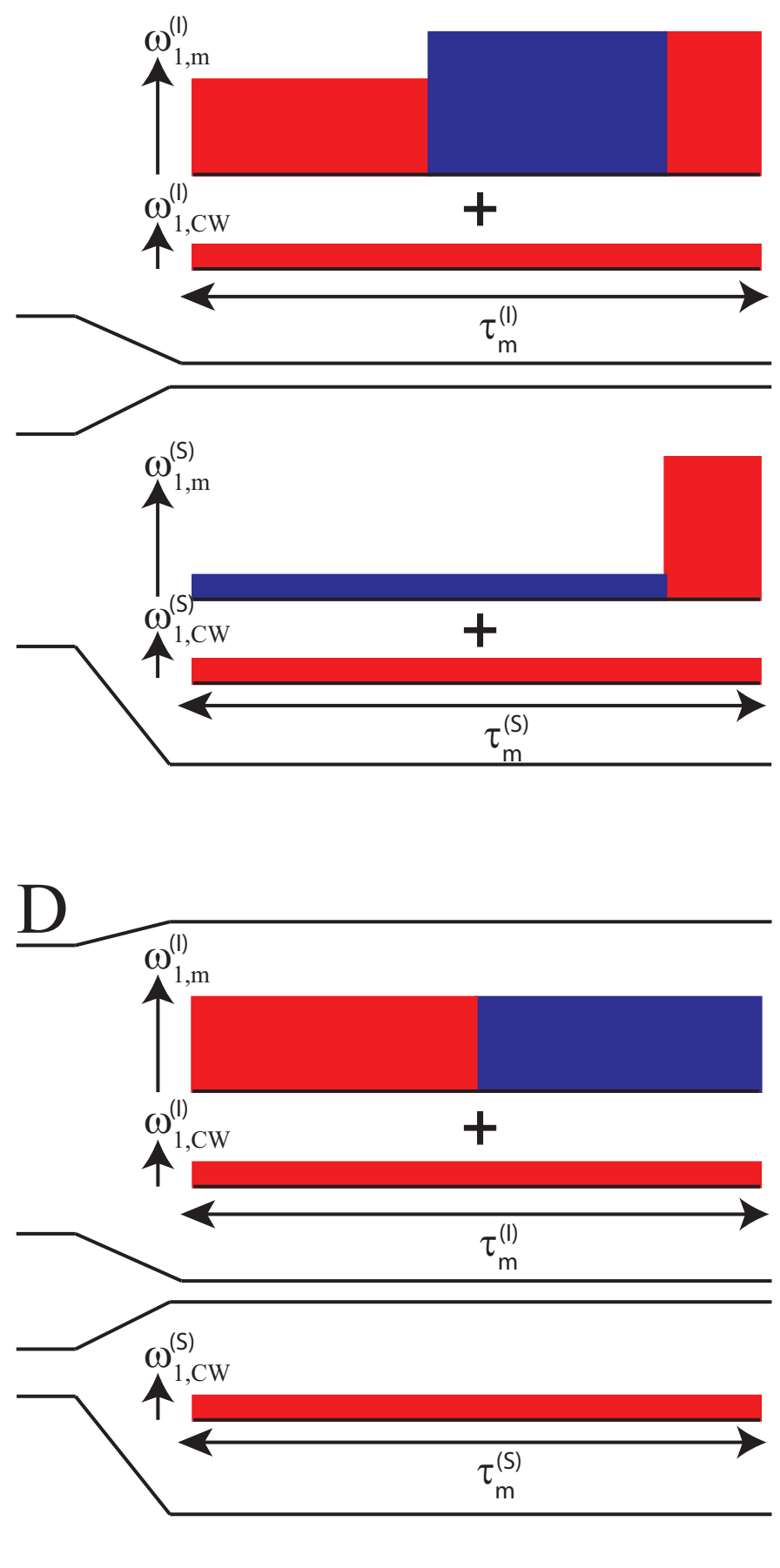

F

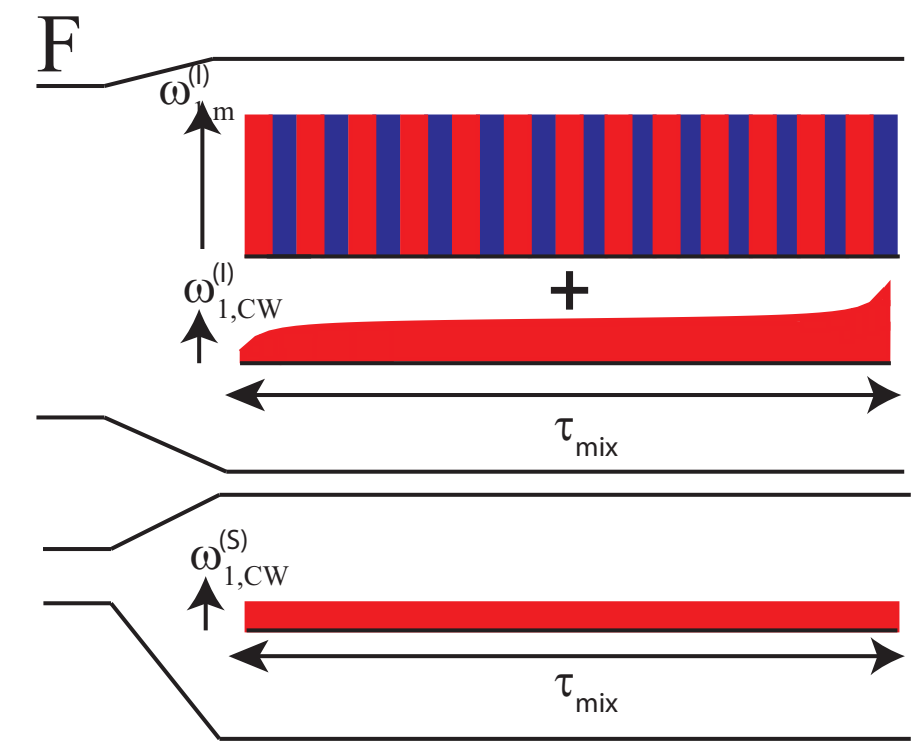



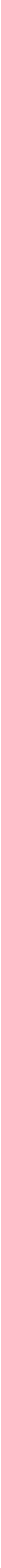

$$
\omega_{1}{ }^{(\Lambda)} / \omega_{\mathrm{r}}
$$
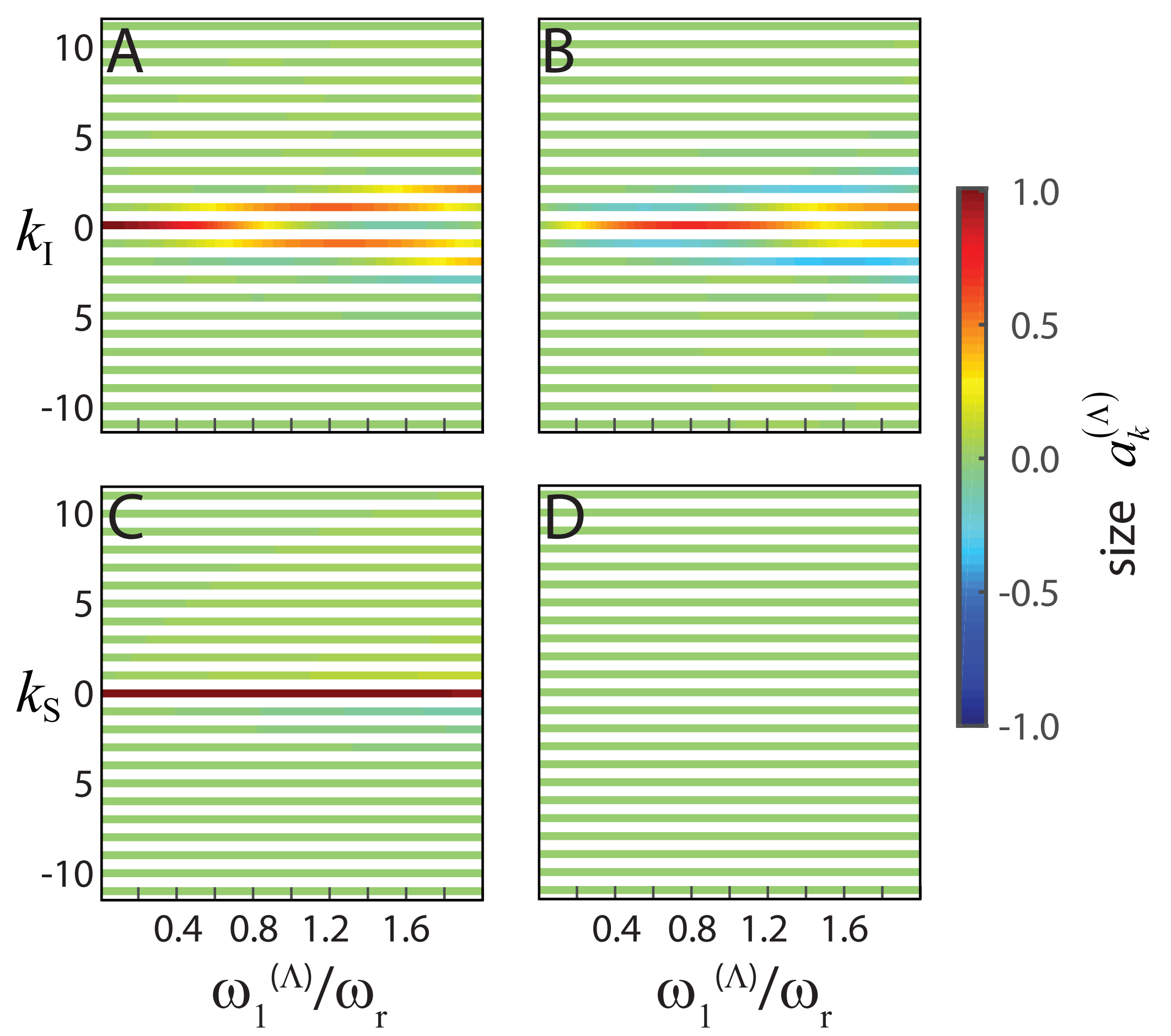


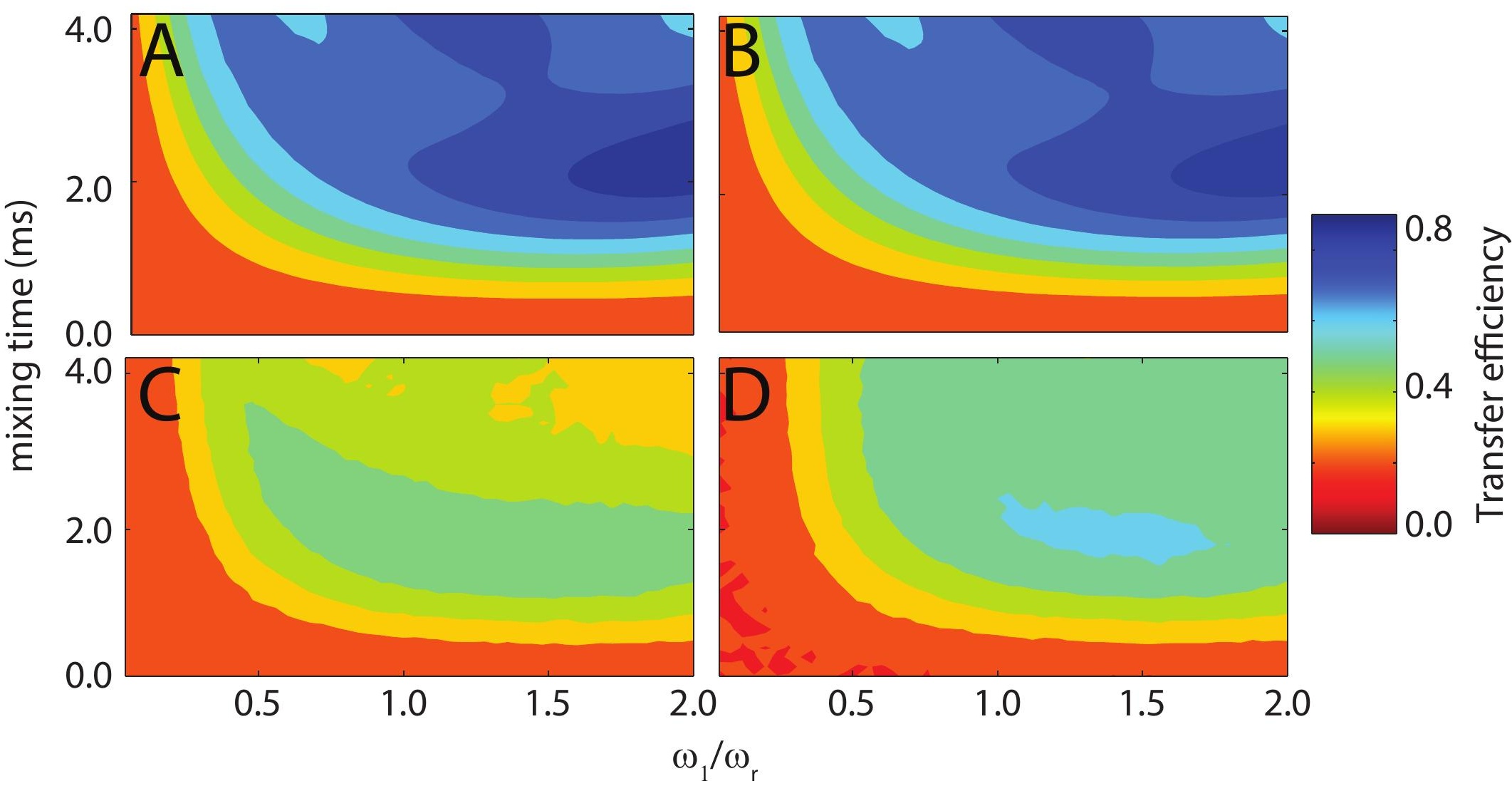


Figure

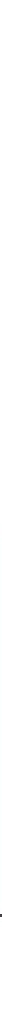




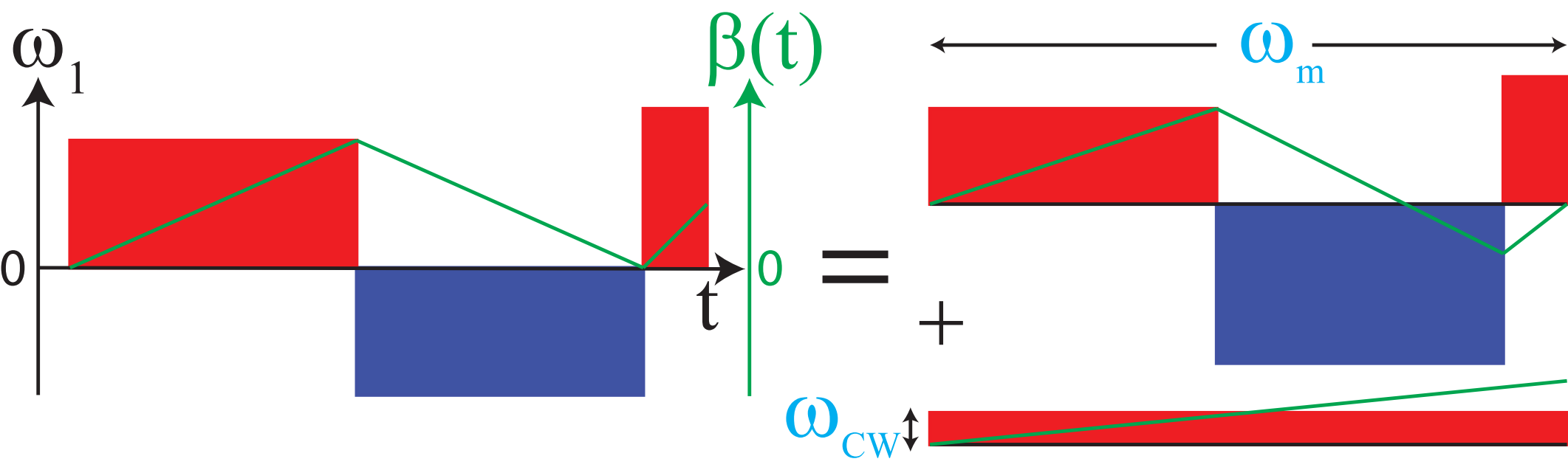

